\title{
Health Information Management: Implications of Artificial Intelligence on Healthcare Data and Information Management
}

\author{
Mary H. Stanfill', David T. Marc² \\ 1 United Audit Systems, Inc., Cincinnati, OH, USA \\ 2 The College of St. Scholastica, Department of Health Informatics and Information Management, \\ Duluth, MN, USA
}

\section{Introduction}

Objective: This paper explores the implications of artificial intelligence (Al) on the management of healthcare data and information and how Al technologies will affect the responsibilities and work of health information management (HIM) professionals. Methods: A literature review was conducted of both peer-reviewed literature and published opinions on current and future use of Al technology to collect, store, and use healthcare data. The authors also sought insights from key HIM leaders via semi-structured interviews conducted both on the phone and by email. Results: The following HIM practices are impacted by Al technologies: 1) Automated medical coding and capturing Al-based information; 2) Healthcare data management and data governance; 3) Patient privacy and confidentiality; and 4) HIM workforce training and education.

Discussion: HIM professionals must focus on improving the quality of coded data that is being used to develop Al applications. HIM professional's ability to identify data patterns will be an important skill as automation advances, though additional skills in data analysis tools and techniques are needed. In addition, HIM professionals should consider how current patient privacy practices apply to Al application, development, and use. Conclusions: Al technology will continue to evolve as will the role of HIM professionals who are in a unique position to take on emerging roles with their depth of knowledge on the sources and origins of healthcare data. The challenge for HIM professionals is to identify leading practices for the management of healthcare data and information in an Al-enabled world.

\section{Keywords}

Artificial Intelligence; Health Information Management; automation; medical coding; health workforce

Yearb Med Inform 2019:56-64

http://dx.doi.org/10.1055/s-0039-1677913
Health information technology has greatly impacted the health information management (HIM) profession. HIM professionals are part of the allied health team and they support efforts to ensure the availability, accuracy, integrity, and security of healthcare data. The digitizing of healthcare data has greatly impacted the responsibilities and work of HIM professionals requiring many to take on more technical roles related to the collection, storage, and use of healthcare data.

The digitizing of healthcare data, as well as advancements in computer processing and data storage, has also enabled the development of advanced algorithms in the form of Artificial Intelligence (AI). As of 2011, the U.S. Agency for Healthcare Research and Quality (AHRQ) had compiled over 17,000 algorithms and computer programs for healthcare evaluation, treatment, and administration [1]. In a recent white paper on AI in Radiology, the Canadian Association of Radiologists stated "In the next 5 years, Canadian radiologists will see more competent AI applications incorporated into PACS workflows, especially for laborious tasks prone to human error such as detection of lung nodules on $\mathrm{x}$-rays or bone metastases on CT." [2].

Multiple factors are driving the development of AI in healthcare. In the United States (U.S.), legislative pressures are mounting to keep pace with other coun- tries regarding AI developments [3]. There are financial pressures on the healthcare industry globally, with increasing demands due to growing and aging population. The industry needs labor-saving technology and techniques to better understand the health of the population while managing the health of a greater number of people and saving money [4]. AI, whether or not it eliminates the need for a person to fill a job, can make the workforce more efficient [5-9]. Accenture estimates that "key clinical health AI applications" can create $\$ 150$ billion in annual savings for the U.S. healthcare economy by 2026 [10]. Even if a fraction of that figure is realized, that is a powerful incentive for adopting AI solutions.

Beyond economic concerns, an additional driver of AI technology is the sheer volume of healthcare data. Healthcare is experiencing an information boom. "The rapid expansion of scientific knowledge and pace of technological development have resulted in an overwhelming sea of data that is difficult to decipher and apply." [11]. Physicians are drowning in data that requires ever more sophisticated interpretation, yet are still expected to perform efficiently. The promise that AI "augments decision making by clinicians by uncovering clinically relevant information hidden in a massive amount of data" [5] is extremely enticing, particularly now, when there are clinician shortages worldwide. The needs-based shortage of healthcare workers globally is estimated at approximately 17.4 million [12]. According 
to the Canadian Association of Radiologists, "...there is evidence that AI can improve the performance of clinicians and that both clinicians and AI working together are better than either alone" [2]. Indeed, AI technology is necessary to achieve the goal of "precision medicine". Precision medicine is an emerging medical model where medical decisions and treatments are tailored to the patient. "Precision medicine presupposes the availability of massive computing power and algorithms that can learn by themselves at an unprecedented rate" [5].

Predictions on when healthcare will experience widespread deployment of disruptive $\mathrm{AI}$ applications vary widely. Though AI is developing rapidly, and there are current and imminent uses of $\mathrm{AI}$ in healthcare, it is still largely immature. According to witnesses who testified before the U.S. Subcommittee on Information Technology of the House Committee on Oversight and Government Reform at a series of hearings on AI held in 2018, "narrow" AI, i.e. systems focused on specific tasks, is commonly used today but more general systems that can work across multiple tasks are underdeveloped [13]. However, given the pace of development, the timeline for AI in healthcare is years, not decades [14].

Presuming AI will eventually be widespread and affordable, there are implications for the management of healthcare data and information in an AI-enabled world which can greatly impact the HIM profession. The purpose of this paper is to describe the results of a literature review and the findings from interviews with key HIM leaders. The paper explores the relationship of the HIM profession and AI, focusing on the following key aspects: 1) Changes in HIM practices for specific HIM use cases, including automated medical coding and management of AI-based information; 2) Changes in management of healthcare data and the need for evolving data practices and data governance; 3) Legal, ethical, and regulatory data challenges; and 4) Changes in the HIM workforce, including foreshadowing new roles and skills that are required. The conclusion presents steps the HIM profession can take now to help advance the development of reliable AI applications and to respond to their use in healthcare.

\section{Changing Health Information Management Practices}

A core responsibility of the HIM profession is ensuring the right information is provided to the right people to enable quality patient care [15]. Increased adoption of AI-enabled applications and more sophisticated use of AI systems by healthcare providers at the point of care have significant implications for HIM practices. These include practical implications both for common HIM processes, such as medical coding, as well as more generally the core HIM responsibility to manage health data and information. This section explores the impact of AI systems on HIM practices for the following use cases:

- Automated medical coding;

- AI-based diagnosis specificity;

- AI-based early detection information.

Each use case includes examples of the anticipated use of AI, discusses the associated impact to current HIM processes and practices, and explores new opportunities and challenges to adapt HIM practices.

\subsection{Automated Medical Coding}

A systematic literature review of published studies evaluating the performance of automated coding and classification systems indicated that automated coding systems have been in use since at least the mid 1990's [16]. Computer-assisted coding (CAC) is the term that refers to the automated generation of medical codes reported on healthcare claims that are derived from clinical documentation. CAC applications have been available since the early 2000's [17] with adoption rates increasing markedly in recent years. According to a report available through Research and Markets, the global market for CAC software is projected to reach $\$ 4.75$ billion by 2022 at a compound annual growth rate of $11.5 \%$ [18]. North America is seeing the largest growth followed by Europe, Asia-Pacific, and the rest of the world.

CAC applications use natural language processing (NLP) to read and interpret clinical documentation in patient health records and suggest applicable diagnosis and procedure codes. Typically, a person reviews the suggested codes to determine the final code selection. This computer-assisted approach to the medical coding process is becoming more common and has been credited with measurable gains in coder productivity [19, 20]. However, productivity impacts vary widely, depending on the specific deployment. Some studies reported a drop in productivity when medical coders were forced to validate, and frequently eliminate, a large number of suggested codes. Still, a Cleveland Clinic study found that CAC increased their coder productivity by over $20 \%$ without reducing quality when suggested codes were reviewed and edited by a medical coder [19]. The referenced Cleveland Clinic study found that $\mathrm{CAC}$ alone, without the intervention of a credentialed coder, however had a lower recall and precision rate.

Adoption of $\mathrm{CAC}$ requires reengineering the medical coding workflow to fully integrate the $\mathrm{CAC}$ tool in the process and gain optimal efficiency [21]. Early adopters of CAC in the U.S. reported that CAC had "...improved medical coding workflows, increased medical coding accuracy, and balanced medical coding resources to focus on more volume and complex cases" [22]. Not all hospitals however have experienced these benefits [23]. Some implementations have failed entirely. Effective implementation of a CAC application requires interfaces to work properly so the application can read all documents relevant for coding. In addition clinical documents must comply with a consistent format dictated by the CAC vendor [24]. And where $\mathrm{CAC}$ has been most effective, a new role has emerged to fine tune the rules and train the system to adapt as the code sets and reporting requirements change.

As the technology advances, and machine learning techniques improve the capabilities of CAC tools, the medical coding workflow will further evolve. A WinterGreen market shares research report released in 2017 stated that as much as $88 \%$ of medical coding in physician offices for billing purposes could occur automatically without human review [25]. This report requires independent validation and more research is needed on the accuracy of these systems to rely on them, but advancements in CAC are poised to 
further augment the medical coding process. Medical coding is a significant responsibility of many HIM professionals currently and this role will continue to evolve.

There are significant opportunities for medical coding professionals as CAC advances to increase coding efficiency. The fully automated coding workflow requires reengineering and a focus on data quality, which medical coders, with their intimate knowledge of the code sets and reporting requirements, are uniquely qualified to address. In addition to assigning or validating codes on complex cases, medical coders could also focus on validating aberrant coded data patterns across large groups of cases. For example, a medical coder has the knowledge to question the use of a code for an acute phase of a condition repeatedly for a patient, when the more likely data pattern would be the acute code followed by codes for the chronic phase or sequela. This code-specific pattern recognition is key in validating accurate reporting for risk-scoring payment methodologies for example. Clearly, HIM professionals' ability to identify data patterns to enhance business intelligence or improve compliance with code reporting requirements will be an important skill as automation advances.

\subsection{Diagnosis Specificity}

AI systems are expected to assist healthcare providers with diagnosis accuracy and specificity. Medical specialties that utilize images for diagnosis (e.g. radiology, pathology, dermatology, ophthalmology) are particularly amendable to AI-aided diagnoses. AI machine learning (ML) is very good at detecting anomalies in images, for example it has been proven effective in detecting lung nodules on a radiologic image $[2,6,9]$ and congenital cataract as well as diabetic retinopathy on ocular image data $[6,26]$. The sensitivity and specificity of deep learning algorithms, in detecting diabetic retinopathy through retinal fundus photographs, for example, are both over $90 \%$, which is "competitive against experienced physicians in the accuracy for classifying both normal and disease cases"[6]. An algorithm that can identify skin cancer by analyzing images of skin lesions has also performed as well as board-certified dermatologists [26, 27]. It has been suggested that what might take an experienced radiologist 30 years of radiology-pathology correlation to master may only take an AI system hours or days to analyze and learn in the future [28].

Code reporting guidelines for using diagnostic test results to add specificity to a diagnosis code vary by country. As AI systems become more adept and are proven reliable in visual diagnosis, the need for physicians to read images may become less necessary, perhaps done only by exception. This change in responsibilities could result in either a decrease in code specificity or less consistency of international diagnosis code data, depending on a country's code reporting guidelines and how the guidelines are adjusted to account for AI. For example, currently in the U.S., "code assignment is based on the documentation by patient's provider (i.e., the physician or other qualified healthcare practitioner legally accountable for establishing the patient's diagnosis)" [29]. U.S. guidelines specifically state that clinically significant "laboratory, x-ray, pathologic, and other diagnostic results" can be used for coding only if the test has been "interpreted by a physician" [29]. In the U.K., the NHS National Clinical Coding Standards, while less explicit than U.S. guidelines, also imply that a physician has to interpret diagnostic test results [30]. In contrast, the Canadian Coding Standards are much more amendable to AI development. Canadian medical coders are directed to use diagnostic results "when they clearly add specificity in identifying the appropriate diagnosis code for conditions documented in the physician/primary care provider notes" [31]. In Canada, there is no specific requirement that the test itself has to be interpreted by a physician. Based on this varying guidance, in the instance where a physician has documented a diagnosis, additional specificity of that diagnosis in images interpreted by an AI system alone (without a physician over-read) would be lost in diagnosis data in the U.S. and possibly the U.K., whereas specificity would not necessarily be lost in Canada.

Medical coding and reporting guidelines and standards will need to be adjusted to account for AI applications. There are mul- tiple points to consider including whether reporting of diagnosis specificity using diagnostic test results should vary depending on the AI application itself. Some method is needed to demonstrate that the AI application meets the same degree of accuracy as physicians. For example, reporting guidelines might depend on whether the AI application is approved or credentialed in some manner. Reporting specificity based on AI results might also depend on whether the AI application is employing supervised verses unsupervised ML techniques. Unsupervised ML is well known for feature extraction, whereas supervised ML, which goes through a training process to determine the best outputs, is more suitable for predictive modeling and is generally considered to provide more clinically relevant results [6]. Thus, the type of $\mathrm{AI}$ and how the AI application is used in the clinical workflow (e.g. whether AI-generated interpretations are validated or certified as equally accurate compared to physicians) could potentially be factors in determining future reporting requirements for diagnosis code specificity.

\subsection{Early Detection Information}

AI systems are expected to assist healthcare providers with early detection of likely or impending conditions, allowing for faster intervention. ML algorithms are proving effective in making inferences about specific health risks and predicting health events. For example, neural network algorithms have proven effective in detecting strokes. Input variables analyzed by the algorithm include stroke-related symptoms such as paresthesia of the arm or leg, acute confusion, vision alteration, problems with mobility, etc. This input data is analyzed to determine the probability of stroke [6]. There are other examples of healthcare data being used to detect and predict future events including hospital readmissions, sepsis, and surgical complications [32-34].

Coding guidelines and standards for reporting suspected or impending conditions also vary from one country to the next. In the U.S., coders are directed to report a condition that remains "suspected and/or impending" at the time of discharge as if it existed or 
was established for a hospital inpatient admission, but not to code it on an outpatient encounter [29]. For outpatient cases the condition is coded to the highest degree of certainty [29]. Similarly, NHS National Coding Standards instructions are to code the diagnosis being "treated or investigated" and an example is given of a "probable myocardial infarction" reported with the code for an acute unspecified myocardial infarction [30]. According to the Canadian Coding Standards however, impending or threatened conditions are coded only when indexed as such in the Canadian version of the 10th revision of the International Statistical Classification of Diseases and Related Health Problems (ICD) ICD-10-CA. In addition, unconfirmed diagnoses in Canada are reported with a specific "Q prefix" to denote the uncertainty associated with the code [31]. This variability and the inability in some countries to qualify reported diagnoses as unconfirmed or uncertain is concerning. Consider for example, if an AI system triggers an alert for suspected sepsis on a patient and the healthcare team takes immediate action, thus intervening and preventing severe sepsis, the coding and reporting of this circumstance may be missed, or inconsistently reported at best. Coding guidelines and standards will need to be revised to capture this sequence of events and support AI developments in early detection of likely or impending conditions. This has broad implications and will require an interdisciplinary team to address the issue fully, including standards developers and members of the healthcare team as well as HIM professionals.

One solution is to capture qualifiers to diagnoses. If the functionality was built into Electronic Health Records (EHRs), the Health Level 7 (HL7) Fast Healthcare Interoperability Resources (FHIR) standard framework could potentially be leveraged to qualify diagnoses [35]. For example, the FHIR code system verification status defines codes as provisional, differential, confirmed, and refuted [35]. A status could potentially be added to reflect AI as the source for a condition or diagnosis. Alternatively, diagnosis qualifiers could also be addressed by the clinical terminology or classification system itself, which is demonstrated in
SNOMED CT. Prefixes, such as Canada's $Q$ prefix, could be defined and appended to ICD codes. Perhaps ICD-11 extension codes could be defined to characterize the degree of certainty of a condition (e.g. unconfirmed, impending) or identify the source for the diagnosis (e.g. clinician, AI system, patient). Again, there are multiple factors to consider. Use of a status, prefix or extension to a code would require some mechanism to ensure it remains linked with the base code. Otherwise data validity would be a major concern. This is the case for example when an "impending" stroke is identified as an actual stroke because the "impending" qualifier was lost. Implications for insurance coverage or payment policy have also to be considered. As the industry continues to refine what is deemed clinically relevant data/information, medical coding standards and guidelines will need to align with such data standards.

\section{Changing Data Management Practices}

Increased adoption of AI-enabled applications, and more sophisticated use of these AI applications by healthcare providers at the point of care, holds practical implications for managing the data. HIM professionals have an opportunity to help develop, implement, and manage the policies and procedures related to governing healthcare data, as well as to support the development, deployment, and assessment of AI models to ensure that the technology can be trusted to improve care and support greater efficiency.

New and more varied data types are generated by AI-enabled applications affecting data practices and data governance. Today, healthcare data is almost entirely encounter-based. Healthcare data is collected during an encounter with specific interaction with a care provider. However, healthcare data also includes streams of data collected remotely and automatically from multiple data sources. As the Internet of Things (IoT) expands further into healthcare, it is necessary to develop infrastructures to support the proliferation and use of these data streams. IoT is a connection of physical objects with network connectivity that are used to collect and exchange data. 'In IoT, Things' refers to a device which is connected to the Internet and transfers the device information to other devices. "The future's data will not be collected solely within the health care setting. The proliferation of mobile sensors will allow physicians of the future to monitor, interpret, and respond to additional streams of biomedical data collected remotely and automatically" [7]. Such applications have been in development for several years. More than five years ago, a blood pressure cuff that connects to a smartphone, and transmits data to a care provider was already available [36]. Devices are also available that measure glucose levels, provide electrocardiogram readings, or even collect measures of people's cognition and emotional health [37]. As wearable sensors improve, they will increasingly allow specific health parameters to be tracked constantly and discreetly. They may replace commonly worn items such as a watch, may be worn under regular clothing, or even built into "smart" clothing [38]. These types of devices would conceivably transmit data back to a healthcare provider, potentially directly into an EHR, which presents numerous challenges. It will be critical to track the source of this data as the accuracy, value, and clinical significance may be uncertain. In addition, today's data practices are entirely oriented toward an episode of care. In AI-enabled healthcare, the underlying organizing schema for health data needs to shift from dates of service to the patient. It may require completely different data architecture to collect, store, process, validate, interpret, and potentially retrieve non-episodic ongoing streams of patient-specific data.

Manogaran and colleagues [39] proposed a framework to support the collection, transfer, and storage of data from multiple data streams. They emphasized that the security of data must occur at numerous stages including during the collection of data from devices, the transfer of data between devices, the storage of data, and during the application and use of the data. Additionally, how the data is received from various streams and integrated into a single system poses a challenge. Data streams may include structured, semi-structured, or unstructured data and for integration to occur 
there is a need for standardization. Initiatives such as International Standard for Metadata Registries (ISO/IEC 11179) aim to support what is referred to as 'semantic interoperability' between data that may be expressed differently across devices and technologies [40]. Semantic interoperability is intended to support the unambiguous exchange of data. One method for standardization is to create globally unique cross-reference identifiers for data elements that are semantically equivalent using eXtensible Markup Language (XML) standards, even though the data elements may have different names [40]. The Open Data Element Framework (O-DEF) was developed by The Open Group and can support the categorization, naming, and indexing of data using a controlled vocabulary that associates data elements with structured unique identifiers so that equivalencies and similarities between data can be easily determined [41]. These identifiers can be the basis of an indexing schema where a data element from one device can be integrated with a data element from another device because they both share the same equivalent content evidenced by the same structured unique identifier. O-DEF works well for collaborating enterprises, but may not serve the purpose of integrating data from disparate systems and organizations. Alternatively, other frameworks such as those from the World Wide Web Consortium (W3C) that focus on data integration of web-based data like RDF (Resource Description Framework), OWL (Ontology Web Language), and SKOS (Simple Knowledge Organization System) may be more useful [42]. Data integration challenges will require an interdisciplinary team to address the issue. HIM professionals can seek to examine how existing information models can be leveraged within an organization to support a data governance framework that accommodates multiple data streams. The utilization of existing vocabularies may serve to accelerate the collection and use of data from non-episodic sources.

An additional challenge is the need for quality healthcare data. ML techniques require substantial amounts of data to ensure algorithms work accurately and are applied appropriately to their targeted goals. "ML algorithms are highly data hungry, often requiring millions of observations to reach acceptable performance levels" [14]. Thus researchers and developers need access to large sets of health data from thousands of patients. The reliability of an AI application is dependent upon the quality of the data that was used to develop and train it. "At its core, $\mathrm{AI}$ is reliant upon data. If the data itself is incomplete, biased, or skewed in some other fashion, the AI system is at risk of being inaccurate" [13]. However, it's widely recognized in the U.S. that data in EHRs and claims databases need "careful curation and processing before they are usable" [14]. Healthcare data are highly heterogeneous, ambiguous, noisy, and incomplete [26]. Data curation (i.e., managing data to make it more useful) requires significant financial investment and without investing resources to support data curation the healthcare industry risks producing ML models based on factually inaccurate data [8]. The adoption of data governance principles can help organizations ensure that the people, processes, and systems involved in $\mathrm{AI}$ initiatives are held accountable for ethical use and deployment, the process is transparent, the result has integrity, the information is protected, the approach is compliant with organizational and legal practices, the technology is available, the method of AI development is retained, and when appropriate the healthcare data is disposed of properly [43]. These principles can help support the use of AI models that minimize the risk to patients, providers, developers, and healthcare organizations.

Evolving data governance principles are necessary and must be a priority for all healthcare organizations. Developing clear, consistent, and standardized policies and procedures for creating and managing current and emerging sources of data is a key enabler to development of $\mathrm{AI}$ applications. Data sources can include EHR data, lab data, imaging data, claims data, various types of master data (e.g., enterprise master patient index), patient-generated data, and metadata as well as a real-time streaming data from medical devices. Several issues need to be managed, such as data sparsity, redundancy, and missing values [26]. Data governance, including data modeling, data standards and definitions, data mapping, data auditing, data quality controls, and data quality management, must keep pace with evolving data types and data uses. For example, data quality management in healthcare organizations today focuses on assuring data is fit for use for the organization's business operations, decision-making and planning. More focus is needed on detecting, assessing, and fixing data defects in a systematic way. Data governance has never been a higher priority in healthcare as it "empowers users to trust the predictions of analytics models in their decision-making because there is certainty that the data and algorithms can be trusted" [44].

As advances in AI enable precision medicine, HIM professionals will need to develop practices to enable precision HIM. Treating all healthcare data and information the same will no longer be practical or efficient in an era of big data. More robust data analytics and processes need to be established to identify data patterns and trends and address data outliers. "Precision medicine attempts to ensure that the right treatment is delivered to the right patient at the right time by taking into account several aspects of patient's data, including variability in molecular traits, environment, EHRs and lifestyle" [26]. Precision HIM attempts to ensure the right data and information is delivered to the right person at the right time by taking into account the data source and the people, processes, and technology that interface with that data to ensure it is used and reused appropriately.

\section{Legal, Ethical, and Regulatory Data Challenges}

The use of healthcare data to develop AI applications has introduced substantial legal, ethical, and regulatory challenges. Patient privacy is a key concern affecting how AI is developed and tested. Development of AI applications may require updates to privacy and confidentiality laws and regulations, which vary widely. In the U.K., protection of health information centers on obtaining explicit consent from the patient in order to share information with any third party that is not in a direct care relationship with the patient. Researchers must apply to the 
Health Research Authority's Confidentiality Advisory Group (CAG) for approval to access confidential patient information without patients' consent [45]. In the U.S., government regulation is less strict. Privacy and confidentiality of protected health information are addressed in the Health Insurance Portability and Accountability Act (HIPAA). HIPAA provides data privacy and security provisions for safeguarding medical information and allows for sharing protected health information without patient consent specifically for the purposes of "treatment, payment and operations"[46]. How the U.K. or U.S. approaches will be interpreted on cases related to data sharing for AI development is largely undetermined. The U.K. consent requirement, and the definition of a "direct care relationship," was challenged in 2017 in a published case study. The case study alleged that a technology company, Google DeepMind, did not have a direct patient care relationship with every patient included in the data shared and thus "held data on millions of Royal Free patients and former patients since November 2015, with neither consent, nor research approval" [47]. This case study underscores the need to examine current privacy laws and regulations to determine how they may apply to AI applications. The U.S. Subcommittee on Information Technology recommends that federal agencies conduct such a review and, where necessary, update existing regulations to account for the addition of AI [13]. HIM professionals are involved with developing and implementing organizational policies regarding privacy and security of health information, training staff, and ensuring compliance. Therefore, with the access and use of health information for the development and deployment of AI models, HIM professionals should explore current privacy practices considering how they might apply to AI applications and how they might be amended to account for AI technology.

In addition to data privacy and protection, another looming legal issue is liability and accountability for the use of AI applications. Questions on who is ultimately liable for patient care decisions based on, or aided by, an AI application are yet to be answered. Should healthcare providers be held fully responsible for decisions suggested by algo- rithms they cannot understand? Will physicians use a system they cannot understand? Can the developer be held responsible? The problem is complicated since the reasoning in an AI application is difficult, often too complex to understand [48]. AI applications evolve and change constantly in unforeseeable ways as they are "learning" from data [9]. Though mechanisms to ensure AI applications are safe and effective are still being formulated, prevailing approaches include an expectation that algorithms can be inspected. "Each algorithm should be able to explain its output" [13]. To advance deployment and acceptance of AI applications, developers will need to be able to produce the algorithm for inspection, support why the algorithm works, and ensure the application can meet expected outcomes in testing or certification procedures. Product master data, which includes data about the components that make up the product, may include information on the algorithm deployed. In the future, individual patient health information may include the algorithm that was applied to the patient's data in order to validate or authenticate healthcare decisions. In addition, there may be a need to audit AI events for reporting purposes. HIM professionals can establish the necessary data governance principles that must be adopted for AI applications to be implemented successfully within healthcare organizations.

Another aspect that deserves attention is the need to balance the financial incentive to make processes more efficient with the ethical and legal uses of health information. For example, the financial motivators to adopt CAC for the sole purpose of coding a higher level of care must be tempered by ethical considerations. HIM professionals involved in the clinical coding process can greatly impact the amount of funding provided to a healthcare organization. Hoyle [49] and Shepherd [50] argued that HIM professionals are positioned as advocates for the ethical use of technology and data. HIM professionals must urge healthcare organizations to consider the ethical frameworks and practice guides not just deemed appropriate for health information professionals, but also for CAC and AI technologies. These activities will provide support for the HIM professionals in healthcare organization to go about the business of "providing the clinical truth in their coding and resisting the perverse incentives" [50]. Therefore, with the access and use of health information for the development and deployment of AI models, HIM professionals should be involved to ensure policies and procedures are being developed, amended accordingly, and followed to account for the influence of AI technology. Although HIM professionals are just beginning to work with AI technology, there has already been notable impacts on the HIM workforce.

\section{Response of the Health Information Management Workforce}

Healthcare technology has greatly impacted the way care is approached and delivered. The digitizing of healthcare data has supported efforts to automate processes that were previously done manually. These processes have inevitably impacted the healthcare workforce, including the HIM profession. There is a greater need for employees that have technical skills to better collect, manage, and use healthcare data. Sandefer and colleagues [51] evaluated data from a workforce survey that yielded responses from 6,475 healthcare professionals that were largely from HIM. The survey asked respondents to rate the percentage of their time they spent on current tasks and how much they anticipate they will spend on these tasks 10 years in the future. The findings of the study suggested that many HIM professionals spent significant time on diagnostic and procedural coding and records processing, but they expected these tasks to decline the most in the future while leadership, teaching, and informatics tasks are expected to increase. Historically, the HIM profession has focused on medical records and coding. However, the profession has evolved into more diverse roles and continues to change with technological advances. Today, many HIM professionals find themselves in diverse roles related to healthcare leadership, teaching, technology, compliance, quality, and informatics [51, 52]. 
In 2018, Sandefer [53] evaluated data from a workforce survey of 274 senior-level professionals within clinical (e.g., hospitals, clinics) and non-clinical (e.g., software vendors, consulting firms) organizations. The goal of the survey was to identify the needed job skills, competencies, and education required by HIM professionals to meet future workforce needs. Seventy-two percent of clinical respondents reported that at least half of coding functions will be automated, and $50 \%$ reported that more than half of the coding functions will be automated in the near future. The paper suggests that the application of natural language processing combined with the quality of voice to text translation will support improvements in extracting meaning from unstructured data, which will greatly revolutionize the healthcare industry.

Automation is also expected to impact the HIM workforce beyond just influencing how diagnostic and procedural coding is approached. Data analytics has been more prolific across the profession. More professionals are moving into roles to evaluate data related to financial, operational, and clinical performance [54]. HIM professionals are becoming more involved with developing solutions for healthcare organizations to better manage and use data. For instance, HIM professionals are actively participating in the development of policies, procedures, and best practices to ensure data are being used ethically and abiding by the required laws when research or data reporting is being adopted [55]. However, in the future, HIM professionals are going to need to be more involved in developing similar policies and procedures to accommodate AI developments. To date, there is very little attention on the needs for data governance to support AI. Without having a workforce to support AI data governance, there will likely be barriers to widespread adoption and use. For instance, past efforts to implement ICU mortality risk scores have been met with reluctance due to a lack of trust in the technology, despite the obvious benefits the technology may serve [56]. By engaging more stakeholders in the development of the technology, including HIM professionals, a culture of acceptance may be achieved by adopting principles of data governance that offer enterprise-wide technology support.
The evolving use of healthcare data for $\mathrm{AI}$ applications is already impacting the roles and responsibilities of HIM professionals. HIM professionals are findings themselves in more leadership roles that govern healthcare data and technology, and more technical roles that involve the access and use of healthcare data for reporting and evaluation purposes [51]. With some tasks being automated, there will likely be continuing opportunity for HIM professionals to take on more tasks that focus on the data collection, validation, analysis, and overall the ethical use of that data. HIM professionals who currently find themselves working in medical coding who embrace automated coding have an opportunity to transition into a role that focuses on data validation to improve the quality of healthcare data. However, to emerge into these roles, these professionals will need technical training related to methods and tools for data storage, acquisition, and analytics. With advancements in technology, many professions are realizing the need for greater competence in computational thinking skills to better translate data into abstract concepts and understand data-based reasoning [57]. Although exact details on how AI technologies will impact the future of HIM are not yet known, current workforce studies suggest that HIM professionals are going to continue to work in more technical roles and will therefore support AI developments and use.

\section{Conclusion}

AI has and will continue to impact the way decisions are made in healthcare. For example, decisions are influenced by ML algorithms that support the prediction of future events, or the use of clinical decision support systems that aid in the detection of anomalies in diagnostic images. The decisions that HIM professionals make are also being impacted. For instance, CAC has supplemented a medical coder's role in selecting diagnostic and procedural codes for healthcare claims. The promise that AI can support a more efficient decision-making process with greater accuracy is certainly a promise worth exploring. HIM professionals should participate in efforts to align medical coding standards and guidelines with evolving data types and standards. In addition, as AI technologies present new and varied types of source data, HIM professionals have an opportunity to influence the development of mechanisms to collect and integrate emerging data types, including non-episodic ongoing streams of patient data and algorithms in product master data for example. The adoption of data standards and vocabularies that support semantic interoperability is part of the solution to the data integration challenge [40] and one that HIM professionals should participate in evaluating and testing.

HIM professionals should also participate in developing the data governance framework within healthcare organizations to establish mechanisms to collect emerging data types from various sources, manage the policies and procedures related to the access and use of data, and develop methods to validate the reliability and impact of AI technology. This includes considering how evolving data structures impact the use and reuse of data and the related policy implications (e.g., data reporting requirements, payment policy). It also includes for example ensuring data governance practices include product master data (e.g., data about the algorithms deployed) to support efforts to audit, inspect, or certify AI applications. These endeavors will require HIM professionals to have the technical knowledge to analyze and monitor AI tools and the necessary technical skills related to collecting and managing healthcare data in AI-enabled healthcare. To acquire such technical skills, HIM professionals may need to seek additional education or training.

There are significant data management practices as well as laws and regulations surrounding the use of healthcare data that have the potential to either impede or enable development of AI applications. HIM professionals can support future AI developments today by increasing data validation efforts and beginning to evaluate relevant policies and processes. HIM professionals should analyze coded data patterns and establish processes to validate coded data across large groups of cases. HIM professionals must focus on detecting, assessing, and fixing data defects in a systematic way in order to improve the quality of current healthcare data 
Health Information Management: Implications of Artificial Intelligence on Healthcare Data and Information Management

that is being used to develop AI applications. Other examples of steps HIM professionals can take now include ensuring the proper laws and regulations are being followed (e.g., ensuring only authorized personnel and technology accesses clinical data), beginning to explore current privacy practices in light of how they may apply to AI applications, and establishing collaborative relationships with data standards developers and informaticists involved in developing AI applications.

Although there is an emphasis on creating policies and procedures to accommodate AI technology, HIM professionals will also find that there are emerging opportunities for careers related to the greater adoption of AI. HIM professionals are well situated to proactively manage and monitor data governance, data sets, and data models related to the implementation and use of AI. AI technologies are not intended to replace healthcare workers, but individuals who are able to adapt to new workflows and processes may replace those who cannot. There are wonderful opportunities for career moves and advancements for those who continue to increase their knowledge of data analytical methods and tools.

The future of AI holds the promise of a more effective and efficient healthcare system built on a strong foundation of reliable and accurate data. HIM professionals manage and support the entire continuum of healthcare data from the collection of the data to the use and disposition of that data. AI technology will continue to evolve and so will the role that HIM professionals would play to support this technology. The challenge for HIM professionals is to identify leading practices to achieve precision HIM and develop practice standards for the management of healthcare data and information in an AI-enabled world.

\section{References}

1. AHRQ. Medal.org (Medical Algorithms): Agency for Healthcare Research and Quality, Innovation Exchange; 2011 [updated November 9, 2011; cited 2018 December 10]. Available from: https:// innovations.ahrq.gov/qualitytools/medalorg-medical-algorithms.

2. Tang A, Tam R, Cadrin-Chênevert A, Guest W, Chong J, Barfett J, et al; Canadian Association of Radiologists. White Paper on Artificial Intelligence in Radiology. Can Assoc Radiol J 2018;69(2):120-35.

3. Trump DJ. Executive order on maintaining American leadership in artificial intelligence. In: President EOot, editor. Federal Register: White House; 2019. p. 3967-72.

4. Noorbakhsh-Sabet N, Zand R, Zhang Y, Abedi V. Artificial Intelligence Transforms the Future of Health Care. Am J Med 2019.

5. Ganapathy K, Abdul SS, Nursetyo AA. Artificial intelligence in neurosciences: A clinician's perspective. Neurol India 2018;66(4):934-9.

6. Jiang F, Jiang Y, Zhi H, Dong Y, Li H, Ma S, et al. Artificial intelligence in healthcare: past, present and future. Stroke Vasc Neurol 2017;2(4):230-43.

7. Johnson KW, Torres Soto J, Glicksberg BS, Shameer K, Miotto R, Ali M, et al. Artificial Intelligence in Cardiology. J Am Coll Cardiol 2018;71(23):2668-79.

8. eHealth Initiative. The Machines are Here: The Influence of AI on Healthcare. EHIDC; 2018.

9. Pesapane F, Volonte C, Codari M, Sardanelli F. Artificial intelligence as a medical device in radiology: ethical and regulatory issues in Europe and the United States. Insights Imaging 2018;9(5):745-53.

10. Collier M, Fu R, Yin L, Christiansen P. Artificial Intelligence (AI): Healthcare's New Nervous System; 2017.

11. Miriovsky BJ, Shulman LN, Abernethy AP. Importance of health information technology, electronic health records, and continuously aggregating data to comparative effectiveness research and learning health care. J Clin Oncol 2012;30(34):4243-8.

12. Mesko B, Hetenyi G, Gyorffy Z. Will artificial intelligence solve the human resource crisis in healthcare? BMC Health Serv Res 2018:18(1):545.

13. Hurd H, Kelly R. Rise of the Machines: Artificial Intelligence and its Growing Impact on U.S. Policy. https://oversight.house.gov/wp-content/ uploads/2018/09/AI-White-Paper-.pdf: Subcommittee on Information Technology Committee on Oversight and Government Reform; 2018.

14. Obermeyer Z, Emanuel EJ. Predicting the Future - Big Data, Machine Learning, and Clinical Medicine. N Engl J Med 2016;375(13):1216-9.

15. Dimick C. Health information management 2025. J AHIMA 2012;83(8):24-31.

16. Stanfill MH, Williams M, Fenton SH, Jender, RA, Hersh WR. A systematic literature review of automated clinical coding and classification systems. J Am Med Inform Assoc 2010;17(6):646-51.

17. Workgroup AC-ACe-H. Delving into computer-assisted coding. J AHIMA 2004;75(10):48A-H.

18. Research and Markets Report. Computer Assisted Coding Market by Software (NLP, Structured input, Integrated Systems), Service, Application (Automated Encoding, Clinical Coding Audit, Management Reporting) - Global Forecast to 2022. Research and Markets; 2017.

19. Dougherty M, Seabold S, White SE Study reveals hard facts on CAC. J AHIMA 2013;84(7):54-6.

20. Crawford M. The truth about computer-assisted coding. J AHIMA 2013;84(7):24-7.

21. Comfort A, D'Amato C, Isom C, Rihanek T, Thomas-Flowers D. Automated Coding Workflow and
CAC Practice Guidance (2013 update). AHIMA Practice Brief 2013.

22. O’Dowd E. HIT Infrastructure: HIT Infrastructure; 2018. [cited 2018]. Available from: https://hitinfrastructure.com/news/computer-assisted-coding-systems-help-orgs-handle-increasing-data.

23. Butler M. Computer-Assisted Coding Reality Check. J AHIMA 2019;90(6):10-3.

24. Eramo LA. JAHIMA 2017. [cited 2019]. Available from: https://journal.ahima.org/2017/06/01/hascac-lived-up-to-its-promise-providers-vendorsweigh-in/.

25. Curtiss ET, Susan E. Computer Assisted Coding: Market Shares, Strategies, and Forecasts, Worldwide 2016 to 2022. WinterGreen Research; 2017.

26. Miotto R, Wang F, Wang S, Jiang X, Dudley JT. Deep learning for healthcare: review, opportunities and challenges. Brief Bioinform 2018;19(6):1236-46.

27. Esteva A, Kuprel B, Novoa RA, Ko J, Swetter SM, Blau HM, et al. Dermatologist-level classification of skin cancer with deep neural networks. Nature 2017;542(7639):115-8.

28. King BF, Jr. Artificial Intelligence and Radiology: What Will the Future Hold? J Am Coll Radiol 2018;15(3 Pt B):501-3.

29. CMS. ICD-10-CM Official Guidelines for Coding and Reporting FY 2019. CDC; 2018.

30. NHS Digital. National Clinical Coding Standards ICD-10 5th Edition. NHS; 2018.

31. Canadian Institute for Health Information. Canadian Coding Standards for Version 2018 ICD-10-CA and CCI. CIHI; 2018.

32. Saqib M, Sha Y, Wang MD. Early Prediction of Sepsis in EMR Records Using Traditional ML Techniques and Deep Learning LSTM Networks. Conf Proc IEEE Eng Med Biol Soc 2018;2018:4038-41.

33. Golas SB, Shibahara T, Agboola S, Otaki H, Sato J, Nakae T, et al. A machine learning model to predict the risk of 30-day readmissions in patients with heart failure: a retrospective analysis of electronic medical records data. BMC Med Inform Decis Mak 2018;18(1):44.

34. Bertsimas D, Dunn J, Velmahos GC, Kaafarani HMA. Surgical Risk Is Not Linear: Derivation and Validation of a Novel, User-friendly, and Machine-learning-based Predictive OpTimal Trees in Emergency Surgery Risk (POTTER) Calculator. Ann Surg 2018;268(4):574-83.

35. HL7. FHIR Release 3 HL7.org2017 [cited 2018 December 10]. Available from: https://hl7.org/ implement/standards/fhir/codesystem-condition-ver-status.html.

36. Cohn J. The Health Tech Report: Robot Will See You Now. The Atlantic 2013(March):59-67.

37. Metz R. The smartphone app that can tell you're depressed before you know it yourself: MIT Technology Review; 2018 [cited 2018 December 10]. Available from: https://www.technologyreview. com/s/612266/the-smartphone-app-that-can-tellyoure-depressed-before-you-know-it-yourself/.

38. Sawh M. The best smart clothing: From biometric shirts to contactless payment jackets: Smart fabrics and garments on sale now or hitting the shops soon Wareable.com2018 [cited 2019 January 14]. Available from: https://www.wareable.com/ smart-clothing/best-smart-clothing. 
39. Manogaran G, Varatharajan R, Lopez D, Kumar PM, Sundarasekar S, Thota C. A new architecture of Internet of Things and big data ecosystem for secured smart healthcare monitoring and alerting system. Future Gener Comput Syst 2018;82:375-87.

40. Bandyopadhyay D, Sen J. Internet of things: Applications and challenges in technology and standardization. Wirel Pers Commun 2011;58(1):49-69.

41. The Open Group. Open Data Element Framework (O-DEF) Version 1.0: The Open Group; 2016 [cited 2019 January 9]. Available from: https:// publications.opengroup.org/c163.

42. W3C. Semantic Web: W3C; 2017 [cited 2019 January 9]. Available from: https://www.w3.org/ standards/semanticweb/.

43. Empel S. The way forward. AHIMA develops information governance principles to lead healthcare toward better data management. J AHIMA 2014;85(10):30-2; quiz 4.

44. eHealth Initiative. Artificial Intelligence in Healthcare. EHIDC; 2018.

45. NHS Health Research Authority. Confidentiality Advisory Group: NHS; 2018 [cited 2018 December 14]. Available from: https://www.hra.nhs.uk/ approvals-amendments/what-approvals-do-i-need/ confidentiality-advisory-group/.

46. U.S. Department of Health \& Human Services. The HIPAA Privacy Rule: HHS; 2015 [cited 2018 December 14]. Available from: https://www.hhs. gov/hipaa/for-professionals/privacy/index.html.
47. Powles J, Hodson H. Google DeepMind and healthcare in an age of algorithms. Health Technol (Berl) 2017;7(4):351-67.

48. Price N. Artificial Intelligence in Health Care: Applications and Legal Issues. The Sci Tech Lawyer 2017;14(1):10-3.

49. Hoyle P. Health Information is central to changes in healthcare: A clinician's view. Health Inf Manag 2018;48(1):48-51.

50. Shepheard J. Ethical leadership and why health information management professionals need to be involved. Commentary on Health Information is central to changes in healthcare: a clinician's view. Health Inf Manag 2018;2018.

51. Sandefer R, Marc D, Mancilla D, Hamada D. Survey Predicts Future HIM Workforce Shifts. J AHIMA 2015;86(7):32-5.

52. Riley M, Robinson K, Prasad N, Gleeson B, Barker E, Wollersheim D, et al. Workforce survey of Australian graduate health information managers: Employability, employment, and knowledge and skills used in the workplace. Health Inf Manag 2019.

53. Sandefer R. New Market Research Supports HIM Reimagined's Call for Professional Adaptation. J AHIMA 2018;89(5):20-3.

54. Marc DT, Robertson J, Gordon L, Green-Lawson ZD, Gibbs D, Dover K, et al. What the Data Say About HIM Professional Trends. J AHIMA 2017;88(5):25-31.

55. Sher ML, Talley PC, Cheng TJ, Kuo KM. How can hospitals better protect the privacy of electronic medical records? Perspectives from staff members of health information management departments. Health Inf Manag 2017;46(2):87-95.

56. Delahanty RJ, Kaufman D, Jones SS. Development and Evaluation of an Automated Machine Learning Algorithm for In-Hospital Mortality Risk Adjustment Among Critical Care Patients. Crit Care Med 2018:46(6):e481-e8.

57. Davies AFD, Gorbis M. Future Work Skills 2020. Institute for the Future, University of Pheonix Research Institute; 2011.

\section{Correspondence to:}

Mary H. Stanfill

1379 N. Gale Mountain Rd.

Pocatello, ID 83204

USA

Tel: + 15134772544

Fax: + 15138739704

E-mail: stanfillm@gmail.com

David T. Marc

1200 Kenwood Ave

Duluth MN 55811

USA

Tel: + 1 218-625-4892

E-mail:dmarc@css.edu 